\title{
Applications of Homotopy Perturbation Transform Method for Solving Newell-Whitehead-Segel Equation
}

\author{
Mohamed Zellal ${ }^{* 1}$, Kacem Belghaba ${ }^{2}$ \\ ${ }^{1}$ Hassiba Benbouali University of Chlef. BP. 151, Hay Essalem, 02000, Algeria. \\ 12 Labratory of Mathematic and Its Applications (LAMAP) \\ University of Oran1, Ahmed Ben Bella, P.O. Box 1524, Oran, 31000, Algeria. \\ 1 E-mail:m.zellal@yahoo.fr, ${ }^{2}$ E-mail:belghaba@yahoo.fr
}

\begin{abstract}
In the present work, we apply an analytical method known as Homotopy Perturbation Transform Method, is used for solving Newell-Whitehead-Segel Equation. Five tests are examined to validate the accuracy of this algorithm. Numerical results show that the proposed method is a more reliable, efficient and convenient one for solving different cases of NewellWhitehead-Segel equation.
\end{abstract}

Keywords: He's polynomials, Homotopy Perturbation Transform Method, Laplace transform method, WhiteheadSegel equation. 2010 MSC No: 35K15, 35A22, 47j30, 49M27.

\section{Introduction}

The Newell-Whitehead-Segel equations have wide applicability in mechanical and chemical engineering, ecology, biology and bio-engineering. Newell-Whitehead-Segel (NWS)equation is solved by using the Adomian decomposition and multi-quadric quasi-interpolation methods [2, an approximate solution to the Newell-Whitehead-Segel equation by the Adomian decomposition method [8, homotopy perturbation method 9], new iterative method [10], He's variational iteration method [11, Laplace Adomian Decomposition Method [12] and a comparison between the reduced differential transform method and the Adomian decomposition method for the Newell-Whitehead-Segel equation [13. In this article, different types of nonlinear Newell- Whitehead-Segel equations are solved by the Homotopy Perturbation Transform Method (HPTM) which is introduced by Khan and Wu 7 by combining the Homotopy perturbation method [1- [5]-6] and Laplace transform method for solving various types of linear and nonlinear partial differential equations. The Newell-Whitehead-Segel equation models written as:

$$
\frac{\partial u}{\partial t}=k \frac{\partial^{2} u}{\partial x^{2}}(x, t)+a u(x, t)-b u^{m}(x, t)
$$

where $u(x, t)$ is a function of the spatial variable $x$ and the temporal variable $t$ with $x \in \mathbb{R}$ and $t \geq 0$. The function $u(x, t)$ may be thought of as the nonlinear distribution of temperature in an infinitely thin and long rod or as the flow velocity of a fluid in an infinitely long pipe with small diameter $a, b$ and $k$ are real numbers with $k>0$ and $m$ is a positive integer.

\footnotetext{
${ }^{*}$ Corresponding author.Mohamed Zellal ${ }^{1} \mathrm{E}$-mail:m.zellal@yahoo.fr
} 2010 Mathematics Subject Classification:35K15, 35A22, 47j30, 49M27. 


\section{Analysis of Homotopy perturbation transform method (HPTM)}

To illustrate the basic idea of (HPTM) [7, we consider a general nonlinear partial differential equation with the initial conditions of the form

$$
\begin{gathered}
D u(x, t)+R u(x, t)+N u(x, t)=g(x, t), \\
u(x, 0)=h(x), u_{t}(x, 0)=f(x),
\end{gathered}
$$

where $D$ is the second order linear differential operator $D=\partial^{2} / \partial t^{2}, R$ is the linear differential operator of less order than $D, N$ represents the general non-linear differential operator and $g(x, t)$ is the source term. Taking the Laplace transform (denoted throughout this paper by $L$ ) on both sides of Eq. (2):

$$
L[D u(x, t)]+L[R u(x, t)]+L[N u(x, t)]=L[g(x, t)] .
$$

Using the differentiation property of the Laplace transform, we have

$$
L[u(x, t)]=\frac{h(x)}{s}+\frac{f(x)}{s^{2}}-\frac{1}{s^{2}} L[R u(x, t)]+\frac{1}{s^{2}} L[g(x, t)]-\frac{1}{s^{2}} L[N u(x, t)] .
$$

Operating with the Laplace inverse on both sides of Eq. (4) gives

$$
u(x, t)=G(x, t)-L^{-1}\left[\frac{1}{s^{2}} L[R u(x, t)+N u(x, t)]\right],
$$

where $G(x, t)$ represents the term arising from the source term and the prescribed initial conditions. Now, we apply the homotopy perturbation method

$$
u(x, t)=\sum_{n=0}^{+\infty} p^{n} u_{n}(x, t)
$$

and the nonlinear term can be decomposed as

$$
N u(x, t)=\sum_{n=0}^{+\infty} p^{n} H_{n}(u)
$$

for some He's polynomials $H_{n}(u) \operatorname{see}([3]-[4])$ that are given by

$$
H_{n}\left(u_{0}, \cdots, u_{n}\right)=\frac{1}{n !} \frac{\partial^{n}}{\partial p^{n}}\left[N\left(\sum_{i=0}^{+\infty}\left(p^{i} u_{i}\right)\right)\right]_{p=0}, n=0,1,2,3, \cdots
$$

Substituting Eqs.(6) and (7) in Eq. (5) we get

$$
\sum_{n=0}^{+\infty} p^{n} u_{n}(x, t)=G(x, t)-p\left(L^{-1}\left[\frac{1}{s^{2}} L\left[R\left(\sum_{n=0}^{+\infty} p^{n} u_{n}(x, t)\right)+\sum_{n=0}^{+\infty} p^{n} H_{n}(u)\right]\right]\right),
$$

which is the coupling of the Laplace transform and the homotopy perturbation method using He's polynomials. Comparing the coefficient of like powers of $p$, the following approximations are obtained

$$
\begin{array}{ll}
p^{0} \quad: \quad u_{0}(x, t)=G(x, t), \\
p^{1}: \quad u_{1}(x, t)=-L^{-1}\left[\frac{1}{s^{2}} L\left[R u_{0}(x, t)+H_{0}(u)\right]\right], \\
p^{2}: \quad u_{2}(x, t)=-L^{-1}\left[\frac{1}{s^{2}} L\left[R u_{1}(x, t)+H_{1}(u)\right]\right], \\
p^{3} \quad: \quad u_{3}(x, t)=-L^{-1}\left[\frac{1}{s^{2}} L\left[R u_{2}(x, t)+H_{2}(u)\right]\right],
\end{array}
$$


Then the solution is

$$
u(x, t)=\lim _{p \rightarrow 1} \sum_{i=0}^{+\infty} p^{i} u_{i}(x, t)=u_{0}(x, t)+u_{1}(x, t)+u_{2}(x, t)+\cdots
$$

For later numerical computation, the expression of $\psi_{n}$ for $n \geq 1$ below denotes the $n$ - term approximation to $u(x, t)$.

$$
\psi_{n}(x, t)=\sum_{i=0}^{n-1} u_{i}(x, t)
$$

Absolute error $=\left|u(x, t)-\psi_{n}(x, t)\right|$.

Remark: Consider the following linear operators and their invers operators.

$$
\begin{aligned}
L_{t} & =\frac{\partial}{\partial t}, \quad L_{x x}=\frac{\partial}{\partial x^{2}}, \quad \frac{\partial^{2} u_{n}}{\partial x^{2}}=\left(u_{n}\right)_{x x}, \\
L^{-1}\left[\frac{1}{s} L\left(t^{n}\right)\right] & =L^{-1}\left[\frac{n !}{s^{n+2}}\right]=\frac{t^{n+1}}{n+1}, \text { for } n=0,1,2,3, \cdots \\
L^{-1}\left[\frac{1}{s} L(.)\right] & =\int_{0}^{t}(.) d s .
\end{aligned}
$$

\section{$3 \quad$ Numerical results}

To illustrate the accuracy and the effectiveness of the present method, several test examples are considered in this section.

Example 1: Consider (NWS) equation if $k=5, a=2, b=-1$ and $m=2$ :

$$
\frac{\partial u(x, t)}{\partial t}=5 \frac{\partial^{2} u(x, t)}{\partial x^{2}}+2 u(x, t)+u^{2}(x, t),
$$

subject to the initial condition

$$
u(x, 0)=\alpha, \quad \alpha \in \mathbb{R} .
$$

Taking Laplace transform both of sides, subject to the initial condition we have

$$
L[u(x, t)]=\frac{\alpha}{s}+\frac{1}{s} L\left[5 u_{x x}+2 u+u^{2}\right] .
$$

Taking Inverse Laplace transform we get

$$
u(x, t)=\alpha+p L^{-1}\left[\frac{1}{s} L\left[5 u_{x x}+2 u+u^{2}\right]\right] .
$$

By homotopy perturbation method, we get

$$
u(x, t)=\sum_{n=0}^{+\infty} p^{n} u_{n}(x, t)
$$

Substitute Eq. 177 in Eq. 16), we get

$$
\sum_{n=0}^{+\infty} p^{n} u_{n}(x, t)=\alpha+p L^{-1}\left\{\frac{1}{s} L\left[\begin{array}{c}
5\left(\sum_{n=0}^{+\infty} p^{n} u_{n}(x, t)\right)_{x x} \\
+2\left(\sum_{n=0}^{+\infty} p^{n} u_{n}(x, t)\right) \\
+\left(\sum_{n=0}^{+\infty} p^{n} H_{n}(u)\right)
\end{array}\right]\right\}
$$


Where $H_{n}$ are He's polynomials that represents the nonlinear terms. The first few components of He's polynomials are given by

$$
\begin{aligned}
& H_{0}(u)=u_{0}^{2} \\
& H_{1}(u)=2 u_{0} u_{1} \\
& H_{2}(u)=2 u_{0} u_{2}+u_{1}^{2} \\
& H_{3}(u)=2 u_{0} u_{3}+2 u_{1} u_{2}
\end{aligned}
$$

By comparing the coefficient of like powers of $p$, we have:

$$
\begin{array}{lll}
p^{0}: & u_{0}(x, t)=\alpha, \\
p^{1}: & u_{1}(x, t)=L^{-1}\left[\frac{1}{s} L\left[5\left(u_{0}\right)_{x x}+2 u_{0}+H_{0}(u)\right]\right] \\
& =\alpha(\alpha+2) t, \\
p^{2}: & u_{2}(x, t)=L^{-1}\left[\frac{1}{s} L\left[5\left(u_{1}\right)_{x x}+2 u_{1}+H_{1}(u)\right]\right] \\
& =\alpha(\alpha+2)(\alpha+1) t^{2}, \\
p^{3}: & u_{3}(x, t)=L^{-1}\left[\frac{1}{s} L\left[5\left(u_{2}\right)_{x x}+2 u_{2}+H_{2}(u)\right]\right] \\
& =2 \alpha(\alpha+2)(\alpha+1)^{2} \frac{t^{3}}{3}, \\
p^{4}: & u_{4}(x, t)=L^{-1}\left[\frac{1}{s} L\left[5\left(u_{3}\right)_{x x}+2 u_{3}+H_{3}(u)\right]\right] \\
& =\alpha(\alpha+2)(\alpha+1)\left(5 \alpha^{2}+10 \alpha+2\right) \frac{t^{4}}{6}
\end{array}
$$

the approximate solution of equation $13-14$ is given as:

$$
\begin{aligned}
u(x, t)=\alpha(\alpha+2) t+ & \alpha(\alpha+2)(\alpha+1) t^{2}+2 \alpha(\alpha+2)(\alpha+1)^{2} \frac{t^{3}}{3} \\
& +\alpha(\alpha+2)(\alpha+1)\left(5 \alpha^{2}+10 \alpha+2\right) \frac{t^{4}}{6}+\cdots
\end{aligned}
$$

the closed form solution will be as follows:

$$
u(x, t)=\frac{2 \alpha e^{2 t}}{2+\alpha\left(1-e^{2 t}\right)} .
$$

which is an exact solution and is same as obtained by [10].

Example 2: Consider (NWS) equation if $k=1, a=2, b=3$ and $m=2$ :

$$
\frac{\partial u(x, t)}{\partial t}=\frac{\partial^{2} u(x, t)}{\partial x^{2}}+2 u(x, t)-3 u^{2}(x, t),
$$

with the initial condition

$$
u(x, 0)=\beta, \quad \beta \in \mathbb{R} .
$$

As before, taking Inverse Laplace transform we find

$$
u(x, t)=\beta+p L^{-1}\left[\frac{1}{s} L\left[u_{x x}+2 u-3 u^{2}\right]\right] .
$$


By applying homotopy perturbation transform method, subject to initial condition we have

$$
\sum_{n=0}^{+\infty} p^{n} u_{n}(x, t)=\beta+p L^{-1}\left\{\frac{1}{s} L\left[\begin{array}{c}
\left(\sum_{n=0}^{+\infty} p^{n} u_{n}(x, t)\right)_{x x} \\
+2\left(\sum_{n=0}^{+\infty} p^{n} u_{n}(x, t)\right) \\
-3\left(\sum_{n=0}^{+\infty} p^{n} u_{n}(x, t)\right)^{2}
\end{array}\right]\right\}
$$

By comparing the coefficient of like powers of $p$, we get:

$$
\begin{array}{ll}
p^{0}: & u_{0}(x, t)=\beta \\
p^{1}: & u_{1}(x, t)=L^{-1}\left[\frac{1}{s} L\left[\left(u_{0}\right)_{x x}+2 u_{0}-3 u_{0}^{2}\right]\right] \\
& =\beta(2-3 \beta) t \\
p^{2} \quad: & u_{2}(x, t)=L^{-1}\left[\frac{1}{s} L\left[\left(u_{1}\right)_{x x}+2 u_{1}-6 u_{0} u_{1}\right]\right] \\
\quad=2 \beta(2-3 \beta)(1-3 \beta) \frac{t^{2}}{2} & \\
p^{3}: & u_{3}(x, t)=L^{-1}\left[\frac{1}{s} L\left[\left(u_{2}\right)_{x x}+2 u_{2}-6 u_{0} u_{2}-3 u_{1}^{2}\right]\right] \\
& =2 \beta(2-3 \beta)\left(27 \beta^{2}-18 \beta+2\right) \frac{t^{3}}{3 !}
\end{array}
$$

Then the series solution is given by

$$
\begin{array}{r}
u(x, t)=\beta+\beta(2-3 \beta) t+2 \beta(2-3 \beta)(1-3 \beta) \frac{t^{2}}{2} \\
+2 \beta(2-3 \beta)\left(27 \beta^{2}-18 \beta+2\right) \frac{t^{3}}{3 !}+\cdots \\
=\frac{-2 \beta e^{2 t}}{-2+3 \beta\left(1-e^{2 t}\right)} .
\end{array}
$$

which is an exact solution and is the same as obtained by [9] and [1]

Example 3: Consider (NWS) equation if $k=1, a=1, b=1$ and $m=2$ :

$$
\frac{\partial u(x, t)}{\partial t}=\frac{\partial^{2} u(x, t)}{\partial x^{2}}+u(x, t)-u^{2}(x, t),
$$

subject to the initial condition

$$
u(x, 0)=\frac{1}{\left(1+e^{\frac{x}{\sqrt{6}}}\right)^{2}} .
$$

By applying aforesaid method subject to initial condition we have

$$
\sum_{n=0}^{+\infty} p^{n} u_{n}(x, t)=\frac{1}{\left(1+e^{\frac{x}{\sqrt{6}}}\right)^{2}}+p L^{-1}\left\{\frac{1}{s} L\left[\begin{array}{c}
\left(\sum_{n=0}^{+\infty} p^{n} u_{n}(x, t)\right)_{x x} \\
+\left(\sum_{n=0}^{+\infty} p^{n} u_{n}(x, t)\right) \\
-\left(\sum_{n=0}^{+\infty} p^{n} u_{n}(x, t)\right)^{2}
\end{array}\right]\right\}
$$


By comparing the coefficient of like powers of $p$, we have:

$$
\begin{aligned}
& p^{0} \quad: \quad u_{0}(x, t)=\frac{1}{\left(1+e^{\frac{x}{\sqrt{6}}}\right)^{2}}, \\
& p^{1} \quad: \quad u_{1}(x, t)=L^{-1}\left[\frac{1}{s} L\left[\left(u_{0}\right)_{x x}+u_{0}-u_{0}^{2}\right]\right] \\
&=\frac{5}{3} \frac{e^{\frac{x}{\sqrt{6}}}}{\left(1+e^{\frac{x}{\sqrt{6}}}\right)^{3} t} \\
& p^{2} \quad: \quad u_{2}(x, t)=L^{-1}\left[\frac{1}{s} L\left[\left(u_{1}\right)_{x x}+u_{1}-2 u_{0} u_{1}\right]\right] \\
& \quad=\frac{25}{36} \frac{e^{\frac{x}{\sqrt{6}}}\left(2 e^{\frac{x}{\sqrt{6}}}-1\right)}{\left(1+e^{\frac{x}{\sqrt{6}}}\right)^{4}} t^{2},
\end{aligned}
$$

Proceeding in a similar manner, we obtain

$$
\begin{gathered}
p^{3} \quad: \quad u_{3}(x, t)=L^{-1}\left[\frac{1}{s} L\left[\left(u_{2}\right)_{x x}+u_{2}-2 u_{0} u_{2}-u_{1}^{2}\right]\right] \\
=\frac{125}{648} \frac{e^{\frac{x}{\sqrt{6}}}\left(4 e^{\frac{2 x}{\sqrt{6}}}-7 e^{\frac{x}{\sqrt{6}}}+1\right)}{\left(1+e^{\frac{x}{\sqrt{6}}}\right)^{5}} t^{3}
\end{gathered}
$$

Then the series solution of equation $23-24$ in closed form is given by

$$
\begin{aligned}
u(x, t)= & \frac{1}{\left(1+e^{\frac{x}{\sqrt{6}}}\right)^{2}}+\frac{5}{3} \frac{e^{\frac{x}{\sqrt{6}}}}{\left(1+e^{\frac{x}{\sqrt{6}}}\right)^{3}} t \\
& +\frac{25}{36} \frac{e^{\frac{x}{\sqrt{6}}}\left(2 e^{\frac{x}{\sqrt{6}}}-1\right)}{\left(1+e^{\frac{x}{\sqrt{6}}}\right)^{4}} t^{2} \\
& +\frac{125}{648} \frac{e^{\frac{x}{\sqrt{6}}}\left(4 e^{\frac{2 x}{\sqrt{6}}}-7 e^{\frac{x}{\sqrt{6}}}+1\right)}{\left(1+e^{\frac{x}{\sqrt{6}}}\right)^{5}} t^{3}+\cdots \\
= & \frac{1}{\left(1+e^{\frac{x}{\sqrt{6}}-\frac{5 t}{6}}\right)^{2}} .
\end{aligned}
$$

As presented by [9] and [1].

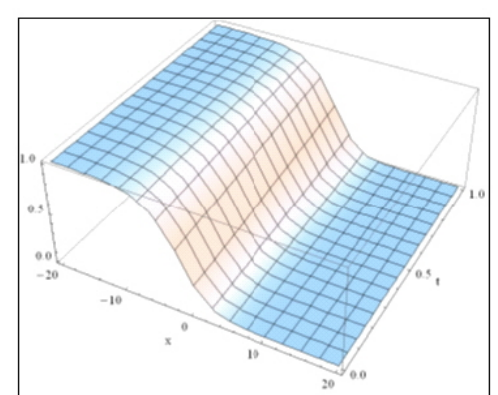

(a)

The behavior of the exact solution (a) in Example 3

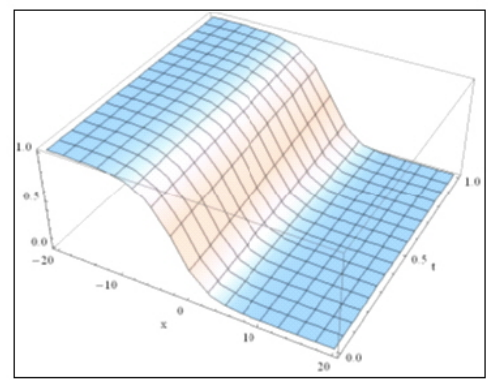

(b)

The behavior of the approximate solution $(b)$ in Example 3

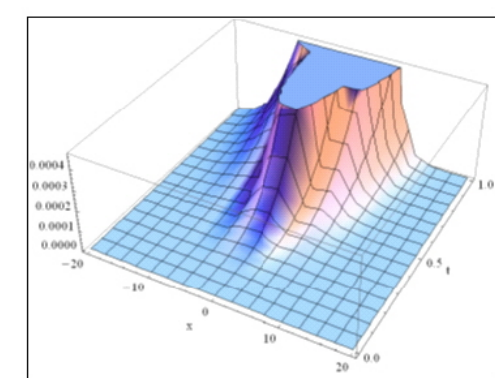

(c)

The behavior of the absolute Error $(c)$ in Example 3 
Table 1: Comparison of the exact solution and $\psi_{4}$ when $t=0.01$ in Example 3.

\begin{tabular}{cccc}
\hline$x$ & Exact value & $\psi_{4}$ & $\left|u-\psi_{4}\right|$ \\
\hline-20 & $9.99476799 \mathrm{E}-1$ & $9.99476800 \mathrm{E}-1$ & $1.11679902 \mathrm{E}-9$ \\
-10 & $9.69673588 \mathrm{E}-1$ & $9.69673631 \mathrm{E}-1$ & $4.29068732 \mathrm{E}-8$ \\
-5 & $7.97929279 \mathrm{E}-1$ & $7.97929085 \mathrm{E}-1$ & $1.93993830 \mathrm{E}-7$ \\
0 & $2.71254811 \mathrm{E}-1$ & $2.71255305 \mathrm{E}-1$ & $4.93486634 \mathrm{E}-7$ \\
5 & $1.53001105 \mathrm{E}-2$ & $1.52999789 \mathrm{E}-2$ & $1.31618083 \mathrm{E}-7$ \\
10 & $3.24044532 \mathrm{E}-4$ & $3.24036591 \mathrm{E}-4$ & $7.94194895 \mathrm{E}-9$ \\
20 & $9.55244894 \mathrm{E}-8$ & $9.55218070 \mathrm{E}-8$ & $2.68256912 \mathrm{E}-12$ \\
\hline
\end{tabular}

Example 4: Consider linear Newell-Whitehead-Segel equation if $k=1, a=1, b=1$ and $m=4$ :

$$
\frac{\partial u(x, t)}{\partial t}=\frac{\partial^{2} u(x, t)}{\partial x^{2}}+u(x, t)-u^{4}(x, t),
$$

subject to the initial condition

$$
u(x, 0)=\frac{1}{\left(1+e^{\frac{3 x}{\sqrt{10}}}\right)^{\frac{2}{3}}} .
$$

By applying the present method (HPTM) subject to initial condition we have

$$
\sum_{n=0}^{+\infty} p^{n} u_{n}(x, t)=\frac{1}{\left(1+e^{\frac{3 x}{\sqrt{10}}}\right)^{\frac{2}{3}}}+p L^{-1}\left\{\frac{1}{s} L\left[\begin{array}{c}
\left(\sum_{n=0}^{+\infty} p^{n} u_{n}(x, t)\right)_{x x} \\
+\left(\sum_{n=0}^{+\infty} p^{n} u_{n}(x, t)\right) \\
-\left(\sum_{n=0}^{+\infty} p^{n} H_{n}(u)\right)
\end{array}\right]\right\}
$$

Where

$$
\sum_{n=0}^{+\infty} p^{n} H_{n}(u)=u^{4}
$$

the first few components of He's polynomials are given by

$$
\begin{aligned}
& H_{0}(u)=u_{0}^{4} \\
& H_{1}(u)=4 u_{0}^{3} u_{1} \\
& H_{2}(u)=2 u_{0}^{2}\left(2 u_{0} u_{2}+u_{1}^{2}\right)+4 u_{0}^{2} u_{1}^{2} .
\end{aligned}
$$


By comparing the coefficient of like powers of $p$,

$$
\begin{aligned}
& p^{0}: u_{0}(x, t)=\frac{1}{\left(1+e^{\frac{3 x}{\sqrt{10}}}\right)^{\frac{2}{3}}}, \\
& p^{1}: u_{1}(x, t)=L^{-1}\left[\frac{1}{s} L\left[\left(u_{0}\right)_{x x}+u_{0}-H_{0}(u)\right]\right] \\
&= \frac{7}{5} \frac{e^{\frac{3 x}{\sqrt{10}}}}{\left(1+e^{\frac{3 x}{\sqrt{10}}}\right)^{\frac{5}{3}} t,} \\
& p^{2}: \quad u_{2}(x, t)=L^{-1}\left[\frac{1}{s} L\left[\left(u_{1}\right)_{x x}+u_{1}-H_{1}(u)\right]\right] \\
& \quad=\frac{49}{100} \frac{e^{\frac{3 x}{\sqrt{10}}}\left(2 e^{\frac{3 x}{\sqrt{10}}}-3\right)}{\left(1+e^{\frac{3 x}{\sqrt{10}}}\right)^{\frac{8}{3}} t^{2},} \\
& p^{3}: \quad u_{3}(x, t)=L^{-1}\left[\frac{1}{s} L\left[\left(u_{2}\right)_{x x}+u_{2}-H_{2}(u)\right]\right] \\
& \quad=\frac{343}{3000} \frac{e^{\frac{3 x}{\sqrt{10}}}\left(4 e^{\frac{6 x}{\sqrt{10}}}-27 e^{\frac{3 x}{\sqrt{10}}}+9\right)}{\left(1+e^{\frac{3 x}{\sqrt{10}}}\right)} t^{\frac{11}{3}},
\end{aligned}
$$

The other components of the (HPTM) can be determined in a similar way. The 4-term approximate solution of equation 25- 26 in is given as:

$$
\begin{aligned}
\psi_{4}(x, t)= & \frac{1}{\left(1+e^{\frac{3 x}{\sqrt{10}}}\right)^{\frac{2}{3}}}+\frac{7}{5} \frac{e^{\frac{3 x}{\sqrt{10}}}}{\left(1+e^{\frac{3 x}{\sqrt{10}}}\right)^{\frac{5}{3}}} t \\
& +\frac{49}{100} \frac{e^{\frac{3 x}{\sqrt{10}}}\left(2 e^{\frac{3 x}{\sqrt{10}}}-1\right)}{\left(1+e^{\frac{3 x}{\sqrt{10}}}\right)^{\frac{8}{3}}} t^{2} \\
& +\frac{343}{3000} \frac{e^{\frac{3 x}{\sqrt{10}}}\left(4 e^{\frac{6 x}{\sqrt{10}}}-27 e^{\frac{3 x}{\sqrt{10}}}+9\right)}{\left(1+e^{\frac{3 x}{\sqrt{10}}}\right)^{\frac{11}{3}}} t^{3} .
\end{aligned}
$$

Hence the exact solution is given as;

$$
u(x, t)=\left\{\frac{1}{2} \tanh \left(\frac{-3}{2 \sqrt{10}}\left(x-\frac{7 t}{\sqrt{ } 10}\right)\right)+\frac{1}{2}\right\}^{\frac{2}{3}}
$$

which is in full agreement with [9] and [1]. 
Table 2: Absolute Error $\left|u-\psi_{4}\right|$ in Example 4 .

\begin{tabular}{cccc}
\hline$x$ & $\left|u-\psi_{4}\right|$ & $\left|u-\psi_{4}\right|$ & $\left|u-\psi_{4}\right|$ \\
\hline & $t=0.1$ & $t=0.3$ & $t=0.5$ \\
\hline 0 & $1.221229 \mathrm{E}-4$ & $3.022038 \mathrm{E}-3$ & $1.245533 \mathrm{E}-2$ \\
0.2 & $1.328287 \mathrm{E}-4$ & $3.415236 \mathrm{E}-3$ & $1.462723 \mathrm{E}-2$ \\
0.4 & $1.352957 \mathrm{E}-4$ & $3.602846 \mathrm{E}-3$ & $1.597269 \mathrm{E}-2$ \\
0.6 & $1.296021 \mathrm{E}-4$ & $3.570756 \mathrm{E}-3$ & $1.636664 \mathrm{E}-2$ \\
0.8 & $1.168616 \mathrm{E}-4$ & $3.333532 \mathrm{E}-3$ & $1.580592 \mathrm{E}-2$ \\
1 & $9.893015 \mathrm{E}-5$ & $2.929772 \mathrm{E}-3$ & $1.440339 \mathrm{E}-2$ \\
\hline
\end{tabular}

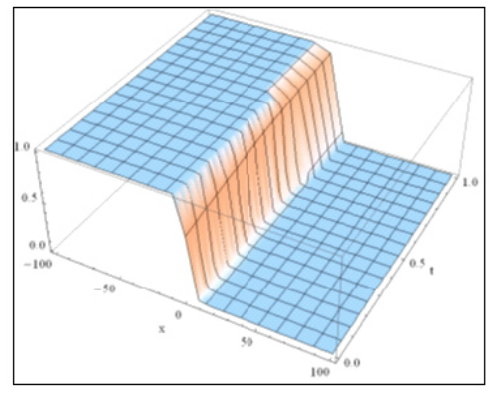

(e)

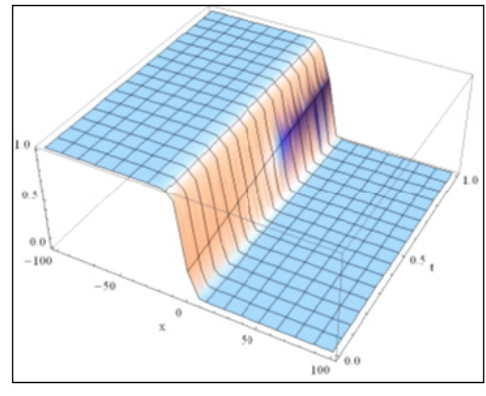

(f)

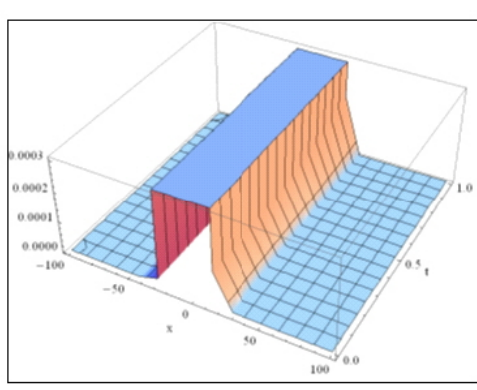

( $g$ )

The behavior of the exact solution $(e)$ in Example $4 \quad$ The behavior of the approximate solution $(f)$ in Example $4 \quad$ The behavior of the absolute Error $(g)$ in Example 4

Example 5: Consider (NWS) equation if $k=1, a=3, b=4$ and $m=1$ :

$$
\frac{\partial u(x, t)}{\partial t}=\frac{\partial^{2} u(x, t)}{\partial x^{2}}+3 u(x, t)-4 u^{3}(x, t)
$$

subject to the initial condition

$$
u(x, 0)=\sqrt{\frac{3}{4}} \frac{e^{\sqrt{6} x}}{e^{\sqrt{6} x}+e^{\frac{\sqrt{6}}{2} x}} .
$$

According to Homotopy Perturbation Transform Method procedures, we have

$$
\sum_{n=0}^{+\infty} p^{n} u_{n}(x, t)=\sqrt{\frac{3}{4}} \frac{e^{\sqrt{6} x}}{e^{\sqrt{6} x}+e^{\frac{\sqrt{6}}{2} x}}+p L^{-1}\left\{\frac{1}{s} L\left[\begin{array}{c}
\left(\sum_{n=0}^{+\infty} p^{n} u_{n}(x, t)\right)_{x x} \\
+3\left(\sum_{n=0}^{+\infty} p^{n} u_{n}(x, t)\right) \\
-4\left(\sum_{n=0}^{+\infty} p^{n} H_{n}(u)\right)
\end{array}\right]\right\}
$$

Where

$$
\sum_{n=0}^{+\infty} p^{n} H_{n}(u)=u^{3}
$$


Table 3: Comparison of the exact solution and $\psi_{4}$ for different time when $x=1$ in Example 5 .

\begin{tabular}{cccc}
\hline$t$ & Exact value & $\psi_{4}$ & $\left|u-\psi_{4}\right|$ \\
\hline 0 & $6.693489 \mathrm{E}-1$ & $6.693489 \mathrm{E}-1$ & 0 \\
0.1 & $7.293730 \mathrm{E}-1$ & $7.292306 \mathrm{E}-1$ & $1.424098 \mathrm{E}-4$ \\
0.2 & $7.736076 \mathrm{E}-1$ & $7.715752 \mathrm{E}-1$ & $2.032386 \mathrm{E}-3$ \\
0.3 & $8.047268 \mathrm{E}-1$ & $7.956462 \mathrm{E}-1$ & $9.080522 \mathrm{E}-3$ \\
0.4 & $8.259108 \mathrm{E}-1$ & $8.007073 \mathrm{E}-1$ & $2.520344 \mathrm{E}-2$ \\
0.5 & $8.400105 \mathrm{E}-1$ & $7.860220 \mathrm{E}-1$ & $5.398846 \mathrm{E}-2$ \\
\hline
\end{tabular}

By comparing the coefficient of like powers of $p$, we find

$$
\begin{aligned}
& p^{0}: u_{0}(x, t)=\sqrt{\frac{3}{4}} \frac{e^{\sqrt{6} x}}{e^{\sqrt{6} x}+e^{\frac{\sqrt{6}}{2} x}}, \\
& p^{1}: \quad u_{1}(x, t)=L^{-1}\left[\frac{1}{s} L\left[\left(u_{0}\right)_{x x}+3 u_{0}-4 u_{0}^{3}\right]\right] \\
&= \frac{9}{2} \sqrt{\frac{3}{4}} \frac{e^{\sqrt{6} x} e^{\frac{\sqrt{6}}{2} x}}{\left(e^{\sqrt{6} x}+e^{\frac{\sqrt{6}}{2} x}\right)^{2}} t, \\
& p^{2} \quad: \quad u_{2}(x, t)=L^{-1}\left[\frac{1}{s} L\left[\left(u_{1}\right)_{x x}+3 u_{1}-12 u_{0}^{2} u_{1}\right]\right] \\
&=\frac{81}{8} \sqrt{\frac{3}{4}} \frac{e^{\sqrt{6} x} e^{\frac{\sqrt{6}}{2} x}\left(-e^{\sqrt{6} x}+e^{\frac{\sqrt{6}}{2} x}\right)}{\left(e^{\sqrt{6} x}+e^{\frac{\sqrt{6}}{2} x}\right)^{3}}, \\
& p^{3}: \quad u_{3}(x, t)=L^{-1}\left[\frac{1}{s} L\left[\left(u_{2}\right)_{x x}+3 u_{2}-4 u_{0}\left(2 u_{0} u_{2}+u_{1}^{2}\right)-8 u_{0} u_{1}^{2}-4 u_{2} u_{0}^{2}\right]\right] \\
&=\frac{243}{48} \sqrt{\frac{3}{4}} \frac{e^{\sqrt{6} x} e^{\frac{\sqrt{6}}{2} x}\left(-4 e^{\sqrt{6} x}+e^{2 \sqrt{6} x}+e^{\sqrt{6} x}\right)}{\left(e^{\sqrt{6} x}+e^{\frac{\sqrt{6}}{2} x}\right)^{4}},
\end{aligned}
$$

Thus, the solution in series form is given by:

$$
\begin{aligned}
u(x, t)= & \sqrt{\frac{3}{4}} \frac{e^{\sqrt{6} x}}{e^{\sqrt{6} x}+e^{\frac{\sqrt{6}}{2}}}+\frac{9}{2} \sqrt{\frac{3}{4}} \frac{e^{\sqrt{6} x} e^{\frac{\sqrt{6}}{2} x}}{\left(e^{\sqrt{6} x}+e^{\frac{\sqrt{6}}{2} x}\right)^{2}} t \\
& +\frac{81}{8} \sqrt{\frac{3}{4}} \frac{e^{\sqrt{6} x} e^{\frac{\sqrt{6}}{2} x}\left(-e^{\sqrt{6} x}+e^{\frac{\sqrt{6}}{2} x}\right)}{\left(e^{\sqrt{6} x}+e^{\frac{\sqrt{6}}{2} x}\right)^{3}} t^{2} \\
& +\frac{243}{48} \sqrt{\frac{3}{4}} \frac{e^{\sqrt{6} x} e^{\frac{\sqrt{6}}{2} x}\left(-4 e^{\sqrt{6} x}+e^{2 \sqrt{6} x}+e^{\sqrt{6} x}\right)}{\left(e^{\sqrt{6} x}+e^{\frac{\sqrt{6}}{2} x}\right)^{4}} t^{3}+\cdots \\
= & \sqrt{\frac{3}{4}} \frac{e^{\sqrt{6} x}}{e^{\sqrt{6} x}+e^{\left(\frac{\sqrt{6}}{2} x-\frac{9}{2} t\right)}} .
\end{aligned}
$$

As presented by [9] 


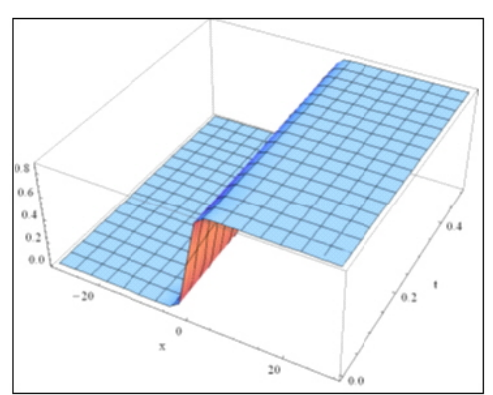

$(h)$

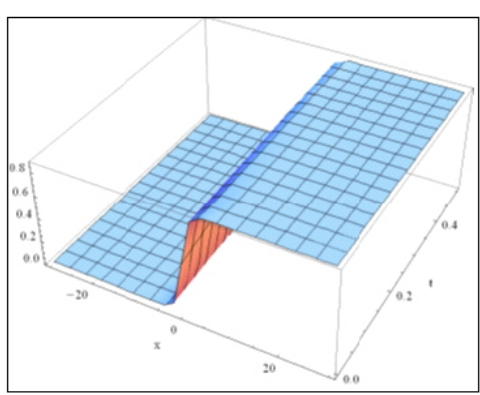

(i)

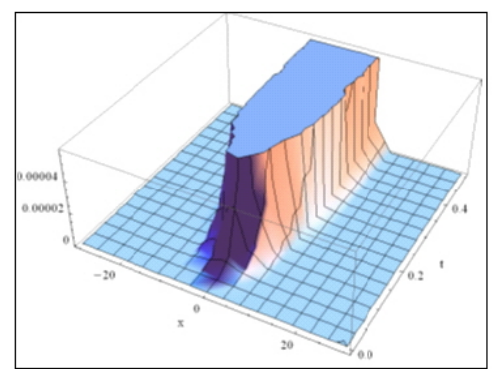

(j)

The behavior of the exact solution $(h)$ in Example 5 The behavior of the approximate solution $(i)$ in Example 5 The behavior of the absolute Error $(j)$ in Example 5

It can be observed through tables (1-3) and figures (1-9) that this method is efficient and accurate for different values of time and place.

\section{Conclusion}

In this paper, the homotopy perturbation transforms method (HPTM) is successfully applied to solve NewellWhitehead-Segel equations. It is worth mentioning that the nonlinear terms can be easily handled by the use of Hes Polynomials. which reduces the computational work as compared to other methods, without using discretization, linearization, or restrictive assumptions, while still maintaining the fast convergence and accuracy of this method. Finally, we conclude that the (HPTM) is considered as a best and accurate method to finding analytic as well as numerical solutions for wider classes of linear and nonlinear differential equations.

ACKNOWLEDGEMENTS.

We thank the editor and the referee for their comments.

\section{References}

[1] J. Biazar, H. Ghazvini, Convergence of the homotopy perturbation method for partial differential equations, Nonlinear Analysis: Real World Applications, 10, (2009), pp.2633-2640.

[2] R. Ezzati, K. Shakibi, Using adomian's decomposition and multi-quadric quasi-interpolation methods for solving Newell-Whitehead equation. Procedia Computer Science, 3, (2011), pp.1043-1048.

[3] A. Ghorbani, J. Saberi-Nadja, perturbation method for calculating adomian polynomials, Inter- national Journal of Nonlinear Sciences and Numerical Simulation, 8(2007), pp.229-232.

[4] A. Ghorbani. adomians polynomials: He polynomials, Chaos Solitons Fractals, 39(2009), pp.1486-1492.

[5] J. H. He. Homotopy perturbation technique. Computer Methods in Applied Mechanics and Engineering, 178, (1999), pp.257-262.

[6] J.H. He. Homotopy perturbation method: a new nonlinear analytical technique, App. Math. Comp, 135(2003), pp.73-79.

[7] Y. Khan and Q. Wu. Homotopy perturbation transform method for nonlinear equations using He's polynomials. Computer and Mathematics with Applications, Vol.61, No.8, (2011), pp.1963-1967.

[8] S. A. Manaa. An Approximate solution to the Newell-Whitehead-Segel equation by the Adomian decomposition method. Raf. J. Comp. Math, Vol.8, No.1, (2011), pp.171-180.

[9] S. S. Nourazar, M. Soori, A. Nazari-Golshan. On the exact solution of Newell-Whitehead-Segel equation using the homotopy perturbation method. Journal of Applied Sciences Research, Vol.5, No.8, (2011), pp.1400-1411.

[10] J. Patade, S Bhalekar. Approximate analytical solutions of Newell-Whitehead-Segel equation using a new iterative method. World Journal of Modelling and Simulation, Vol.11, No.2, (2015), pp.94-103.

[11] A. Prakash, M. Kumar. He's Variational Iteration Method for the Solution of Nonlinear Newell-Whitehead-Segel Equation. Journal of Applied Analysis and Computation, Vol.6, No.3, (2016), pp.738-748.

[12] P. Pue-on , Laplace Adomian Decomposition Method for Solving Newell- Whitehead-Segel Equation. Applied Mathematical Sciences, Vol. 7, No. 132, (2013) 65936600 
[13] A. Saravanan, N. Magesh. A comparison between the reduced differential transform method and the Adomian decomposition method for the Newell-Whitehead-Segel equation. Egyptian Math. Soc, 21, (2013), pp.259-265. 